\title{
CORRIGENDUM
}

\section{Breastfeeding leads to lower blood pressure in 7-year-old Japanese children: Tohoku Study of Child Development}

Miki Hosaka, Kei Asayama, Jan A Staessen, Takayoshi Ohkubo, Katsuhisa Hayashi, Nozomi Tatsuta, Naoyuki Kurokawa, Michihiro Satoh, Takanao Hashimoto, Takuo Hirose, Taku Obara, Hirohito Metoki, Ryusuke Inoue, Masahiro Kikuya, Kunihiko Nakai, Yutaka Imai and Hiroshi Satoh

Hypertension Research (2013) 36, 184; doi:10.1038/hr.2012.203

Correction to: Hypertension Research (2013) 36, 117-122;

doi:10.1038/hr.2012.128; published online 6 September 2012

The authors of the above article noticed an error in publication of this paper (online publication 6 September 2012). HBP values in its abstract section were wrong. Article with corrected values now appears in this issue. The online html and pdf versions have also been rectified. 